\title{
40 years on: have we finally got a vaccine for Pseudomonas aeruginosa?
}

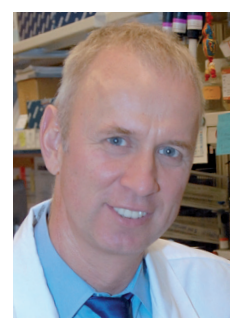

"What type of immunity needs to be induced by an active $P$. aeruginosa vaccine? The answer to this question, critical for a reasonable vaccine design, has not yet been completely answered."

\section{Stefan Worgall}

Department of Genetic Medicine \& Department of Pediatrics, Weill Cornell Medical College, 1300 York Avenue, New York, NY 10065, USA = stw2006@med.cornell.edu

Unfortunately, the quick answer to this question remains no. This does not mean that there has not been substantial progress in the development of a vaccine against this challenging organism. Numerous vaccine candidates have been developed over the past 40 years and a myriad of promising preclinical data have been generated for many of these. Despite these efforts, clinical trials have so far not resulted in a marketable product. A lot has been learned, however, particularly regarding host defenses, mucosal immunity and the biology of the organism. This is essential to better understand host responses that must be induced to protect against Pseudomonas aeruginosa, and will ultimately lead to an efficacious vaccine.

Why has the development of a vaccine against $P$. aeruginosa been so challenging? And do we still need a vaccine? The answer to the latter question is yes. Despite major advances in antimicrobial therapies, the high level of antimicrobial resistance of $P$. aeruginosa remains a major problem for its eradication with antibiotics [1]. This could be alleviated by a vaccine. In answer to the former question, the development of a vaccine against $P$. aeruginosa has been challenging for numerous reasons. Factors both on the pathogen side, as well as in the specific host environment of the individuals at risk, are responsible.

First, there is the ubiquitous nature of this opportunistic pathogen, which is often present in water reservoirs and soil. Most of us are frequently exposed to $P$. aeruginosa. Innate defense mechanisms, particularly in the respiratory tract, usually do not allow $P$. aeruginosa to colonize and infect healthy individuals. This situation is different when host defenses are impaired, and so defines the populations that would benefit from a vaccine against $P$. aeruginosa. Impairment of local defense mechanisms, particularly in the respiratory tract or of systemic host defenses, is the main reason $P$. aeruginosa poses a health problem. Impaired host defenses are also a challenge for effective vaccination. Impaired respiratory clearance mechanisms are present in patients with bronchiectasis, a condition that predisposes to colonization and infection with $P$. aeruginosa. The host environment in the respiratory tract is particularly complex for individuals with cystic fibrosis (CF), who constitute one of the main target populations that benefit from a vaccine against $P$. aeruginosa. The milieu in the respiratory tract in CF favors colonization by bacterial pathogens, specifically $P$. aeruginosa. The reasons for this are still incompletely understood. The CF milieu favors mutations that lead not only to antibiotic resistance but also to mucoid forms and, finally, to the development of biofilms. This affects accessibility to clearance by immune mechanisms, which also includes vaccine-induced immunity. The CF milieu directly and indirectly affects innate host defenses, with effects such as volume and electrolyte imbalance, an altered mucus barrier, impairment of phagocytosis, inflammatory dysregulation and increased bacterial adherence [2]. A vaccine against $P$. aeruginosa in CF would, thus, be most useful before colonization is established. This is not a trivial task, as colonization with $P$. aeruginosa often starts in infancy, an age when it is not easy to induce robust and longlasting immunity against extracellular bacterial pathogens by vaccination. Furthermore, it is unknown whether local immune dysfunction in CF that is already present at this age may affect the efficacy of a vaccine.

Another group of patients who are at risk for severe $P$. aeruginosa infection are those with impaired systemic immunity, including patients with severe burns. Most bloodstream infections

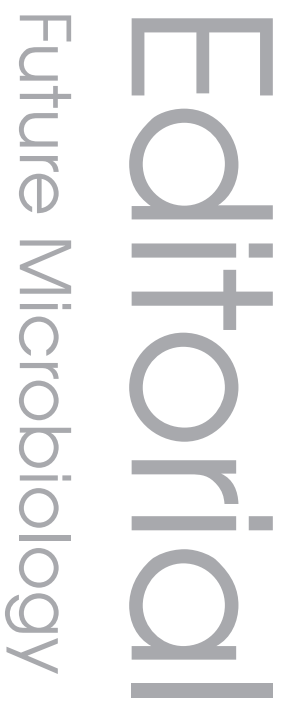

\section{Keywords}

- cystic fibrosis

- Pseudomonas aeruginosa

respiratory infection

- respiratory mucosal

immunity = vaccine

\section{Future Medicine part of}


in burn patients are now caused by Grampositive pathogens, in particular Staphyloccus aureus. However, $P$. aeruginosa still remains the most common Gram-negative pathogen causing life-threatening systemic infections [3]. In $\mathrm{CF}$, systemic infections with $P$. aeruginosa generally do not occur. These two target groups for a vaccine may benefit from different vaccine strategies. For CF, other forms of bronchiectasis or other chronic risk for pulmonary infection with intact systemic immunity, active immunization to establish robust and long-lasting mucosal immunity in order to prevent colonization seems the most effective strategy. To prevent infections in patients with systemic immune suppression in a more acute setting, including severe burns, passive immunization strategies (e.g., antibodies) should be most practical, as active immunization usually requires weeks to months to be protective.

Most efforts in $P$. aeruginosa vaccine development over the past 40 years have focused on developing platforms for active vaccination. Fewer passive immunotherapies have been developed and tested in clinical trials. So why did none of these efforts lead to clinically marketable vaccine? Numerous $P$. aeruginosa antigens and delivery systems were tested as vaccine candidates [4]. The most promising $P$. aeruginosa vaccine antigens identified thus far include the lipopolysaccharide (LPS) O-antigens, outer membrane proteins, flagella and $\operatorname{PcrV}$, and antigens of the type III secretion system. Others that have not been further developed include pili, mucoid exopolysaccharides, exotoxin A and proteases. Each of these antigens induced protective immunity in preclinical models, which most often were studies in rodents. However, testing vaccine candidates in rodents with a healthy immune system does not mimic the complex immune environment of the populations at risk. As outlined above, numerous parts of the innate immune system are altered in CF and, unfortunately, there has been no good rodent model available that mimics human CF lung disease.

LPS, the major component of the outer membrane of $P$. aeruginosa, contains highly variable long chain polysaccharides (O-antigen). LPS O-antigens have been the most widely characterized and investigated vaccine antigens during the last 40 years. LPS antigens were the first to be used for a $P$. aeruginosa vaccine and have also been trialed towards developing passive immunotherapy. These antigens probably induce the highest level of opsonophagocytic and protective immunity, but unfortunately differ for each serotype. The initial enthusiasm in clinical trials in CF and burn patients could not be confirmed in larger studies and the development of vaccines for this antigen have stalled. Flagellin is a more conserved antigen and only two major forms exist. In a large trial in CF patients, a flagellin vaccine induced robust humoral immunity. Surprisingly, $P$. aeruginosa infection with a new flagellin antigen occurred in vaccinated individuals [5]. The most conserved antigens are within the outer membrane proteins, which makes them attractive candidates. No large clinical trials have yet been conducted, although smaller clinical trials have been promising - in particular, in inducing mucosal immunity in the respiratory tract [6]. Conserved antigens, such as ORMs are also promising candidates for genetic vaccine platforms, which can be tailored to induce more favorable mucosal immunity [7].

So far, the major effort to develop passive immunotherapies has included a trial of hyperimmune immunoglobulin LPS O-antigenspecific IgG. This strategy failed, probably due to strain diversity or antibody-mediated mechanisms not being protective [8]. Monoclonal antibodies against alginate, flagella, pilus, PcrV and exotoxin A have been generated [9], however, they have yet to be tested in larger clinical trials. I would anticipate that these efforts will further increase in the coming years. Monoclonal antibodies have been generated against other infectious diseases and this approach makes the most sense to protect immunosuppressed patients.

What type of immunity needs to be induced by an active $P$. aeruginosa vaccine? The answer to this question, critical for a reasonable vaccine design, has not yet been completely answered. Compared with decade-long vaccine efforts against other pathogens, there has only been a limited number of larger clinical trials to provide information on what part of the immune response induced by the vaccine actually provides protective immunity. It is particularly unclear what type of mucosal response is required to prevent colonization of the respiratory tract in CF. The classic thinking entailed that high levels of opsonophagocytic antibodies should be sufficient. More than two decades ago it was already found that for CF patients, antibodies against alginate have been shown to correlate with less chronic mucoid $P$. aeruginosa infection [10]; on the other hand, there are high levels of systemic antibodies in CF patients colonized with $P$. aeruginosa. It now seems that antibodies alone are not sufficient to protect against lung 
infection. In addition to CD4 cellular immunity, it now appears likely that multiple cellular and humoral parts of the immune system must be activated to provide protection. For mice these include neutrophils [11], complement [12], NKT cells [13] and Th17 cells [14]. New promising approaches focus on Th17 immunity, which seems to be particularly important for bacterial lung infections in CF [15]. Th17-mediated mechanisms provide antibody-independent protective immunity against bacterial pathogens in mice [16], including $P$. aeruginosa [14]. Using reverse genetics approaches, Th17-stimulating $P$. aeruginosa antigens, including OprL or lesswell-studied proteins of the $P$. aeruginosa type III secretion system, such as PopB, have recently been identified as new antigens stimulating a strong Th17 response [17].

So where do we stand now? I think the real challenge for the development of a vaccine that is effective in CF, is the preclinical assessment for efficacy in relevant models before engaging in clinical trials. The recently developed new CF animal models, pig and ferret, may have a contribution here, however these are not yet widely and readily available [18]. A better understanding of the critical factor of mucosal immune responses in CF is critical, and there should be a focus on mucosal immunization strategies [19]. Furthermore, the rapidly evolving field of unraveling human microbiomes, including the complex microbiome in the CF lung, will likely provide cues and new insight for the better design of vaccines and, potentially, the identification of new antigens. At the same time, immunotherapies with improved antibody strategies may be able to provide protection. So, hopefully, we won't have to wait another 40 years to be able to effectively prevent infections with $P$. aeruginosa for patients at risk.

\section{Financial \& competing interests disclosure \\ Studies in $S$ Worgall's laboratory on a Pseudomonas aeruginosa vaccine were supported by grants from $\mathrm{NIH}$ UO1 AI069032 and RO1 AI72238. The author has no other relevant affiliations or financial involvement with any organization or entity with a financial interest in or financial conflict with the subject matter or mate- rials discussed in the manuscript apart from those disclosed. \\ No writing assistance was utilized in the production of this manuscript.}

\section{References}

1. Sun HY, Fujitani S, Quintiliani R et al. Pneumonia due to Pseudomonas aeruginosa: part II: antimicrobial resistance, pharmacodynamic concepts, and antibiotic therapy. Chest 139, 1172-1185 (2011).

2. Cohen TS, Prince A. Cystic fibrosis: a mucosal immunodeficiency syndrome. Nat. Med. 18(4), 509-519 (2012).

3. Shupp JW, Pavlovich AR, Jeng JC et al. Epidemiology of bloodstream infections in burn-injured patients: a review of the national burn repository. J. Burn Care Res. 31, 521-528 (2010).

4. Sharma A, Krause A, Worgall, S. Recent developments for Pseudomonas vaccines. Hum. Vac. 7, 999-1011 (2011).

5. Doring G, Meisner C, Stern M. A doubleblind randomized placebo-controlled Phase III study of a Pseudomonas aeruginosa flagella vaccine in cystic fibrosis patients. Proc. Natl Acad. Sci. USA 104, 11020-11025 (2007).

6. Sorichter S, Baumann U, Baumgart A et al. Immune responses in the airways by nasal vaccination with systemic boosting against Pseudomonas aeruginosa in chronic lung disease. Vaccine 27, 2755-2759 (2009).

7. Krause A, Whu WZ, Xu Y et al. Protective anti-Pseudomonas aeruginosa humoral and cellular mucosal immunity by AdC7-mediated expression of the $P$. aeruginosa protein OprF. Vaccine 29, 2131-2139 (2011).

8. Donta ST, Peduzzi P, Cross AS et al. Immunoprophylaxis against klebsiella and Pseudomonas aeruginosa infections. The federal hyperimmune immunoglobulin trial study group. J. Inf. Dis. 174, 537-543 (1996).

9. Pier GB, Boyer D, Preston M et al. Human monoclonal antibodies to Pseudomonas aeruginosa alginate that protect against infection by both mucoid and nonmucoid strains. J. Immunol. 173, 5671-5678 (2004).

10. Pier GB, Saunders JM, Ames P et al. Opsonophagocytic killing antibody to Pseudomonas aeruginosa mucoid exopolysaccharide in older noncolonized patients with cystic fibrosis. N. Engl. J. Med. 317, 793-798 (1987).

11. Koh AY, Priebe GP, Ray C et al. Inescapable need for neutrophils as mediators of cellular innate immunity to acute Pseudomonas aeruginosa pneumonia. Infec. Immun. 77, 5300-5310 (2009).

12. Mueller-Ortiz SL, Drouin SM, Wetsel RA. The alternative activation pathway and complement component $\mathrm{C} 3$ are critical for a protective immune response against Pseudomonas aeruginosa in a murine model of pneumonia. Infec. Immun. 72, 2899-2906 (2004).
13. Nieuwenhuis EE, Matsumoto T, Exley A et al. CD1d-dependent macrophage-mediated clearance of Pseudomonas aeruginosa from lung. Nat. Med. 8, 588-593 (2002).

14. Priebe GP, Walsh RL, Cederroth TA et al. IL-17 is a critical component of vaccineinduced protection against lung infection by lipopolysaccharide-heterologous strains of Pseudomonas aeruginosa. J. Immunol. 181, 4965-4975 (2008).

15. Tan HL, Regamey N, Brown S et al. The Th17 pathway in cystic fibrosis lung disease. Am. J. Resp. Crit. Care Med. 184, 252-258 (2011).

16. Chen K, McAleer JP, Lin Y et al. Th17 cells mediate clade-specific, serotype-independent mucosal immunity. Immunity 35, 997-1009 (2011).

17. Wu W, Huang J, Duan B et al. Th17stimulating protein vaccines confer protection against Pseudomonas aeruginosa pneumonia. Am. J. Resp. Crit. Care Med. 186, 420-427 (2012).

18. Fisher JT, Zhang Y, Engelhardt JF. Comparative biology of cystic fibrosis animal models. Methods Mol. Biol. 742, 311-334 (2011).

19. Chen K, Cerutti A. Vaccination strategies to promote mucosal antibody responses. Immunity 33, 479-491 (2010). 